\title{
Precision of field triage in patients brought to a trauma centre after introducing trauma team activation guidelines Marius Rehn*1,2, Torsten Eken ${ }^{3}$, Andreas Jorstad Krüger ${ }^{1,4}$, Petter Andreas Steen ${ }^{2,5}$, Nils Oddvar Skaga ${ }^{1,6}$ and Hans Morten Lossius ${ }^{1}$
}

\begin{abstract}
Address: ${ }^{1}$ Department of Research and Development, Norwegian Air Ambulance Foundation, Drobak, Norway, ${ }^{2}$ Faculty of Medicine, Faculty Division Ulleval University Hospital, University of Oslo, Norway, ${ }^{3}$ Department of Anaesthesiology, Aker University Hospital, Oslo, Norway, ${ }^{4}$ Department of Anaesthesiology and Emergency Medicine, St. Olav University Hospital, Trondheim, Norway, ${ }^{5}$ Prehospital division, Ulleval University Hospital, Oslo, Norway and 'Department of Anaesthesiology, Division of Emergency Medicine, Ulleval University Hospital, Oslo, Norway

Email: Marius Rehn* - marius.rehn@snla.no; Torsten Eken - torsten.eken@medisin.uio.no; Andreas Jorstad Krüger - andreas.kruger@snla.no; Petter Andreas Steen - p.a.steen@medisin.uio.no; Nils Oddvar Skaga - noskaga@online.no; Hans Morten Lossius - hans.morten.lossius@snla.no * Corresponding author
\end{abstract}

Published: 9 January 2009

Scandinavian Journal of Trauma, Resuscitation and Emergency Medicine 2009, 17:1

This article is available from: http://www.sjtrem.com/content/I7/I/I

(c) 2009 Rehn et al; licensee BioMed Central Ltd.

This is an Open Access article distributed under the terms of the Creative Commons Attribution License (http://creativecommons.org/licenses/by/2.0), which permits unrestricted use, distribution, and reproduction in any medium, provided the original work is properly cited.

\begin{abstract}
Background: Field triage is important for regional trauma systems providing high sensitivity to avoid that severely injured are deprived access to trauma team resuscitation (undertriage), yet high specificity to avoid resource overutilization (overtriage). Previous informal trauma team activation (TTA) at Ulleval University Hospital (UUH) caused imprecise triage. We have analyzed triage precision after introduction of TTA guidelines.
\end{abstract}

Methods: Retrospective analysis of 7 years (200I-07) of prospectively collected trauma registry data for all patients with TTA or severe injury, defined as at least one of the following: Injury Severity Score (ISS) > 15, proximal penetrating injury, admitted ICU > 2 days, transferred intubated to another hospital within 2 days, dead from trauma within 30 days. Interhospital transfers to $\mathrm{UUH}$ and patients admitted by non-healthcare personnel were excluded. Overtriage is the fraction of TTA where patients are not severely injured (I-positive predictive value); undertriage is the fraction of severely injured admitted without TTA (I-sensitivity).

Results: Of the 4659 patients included in the study, 2221 (48\%) were severely injured. TTA occurred 4440 times, only 2002 of which for severely injured (overtriage 55\%). Overall undertriage was $10 \%$. Mechanism of injury was TTA criterion in I 508 cases (34\%), of which only 392 were severely injured (overtriage 74\%). Paramedic-manned prehospital services provided $66 \%$ overtriage and $17 \%$ undertriage, anaesthetist-manned services $35 \%$ overtriage and $2 \%$ undertriage. Falls, high age and admittance by paramedics were significantly associated with undertriage. A Triage-Revised Trauma Score (RTS) < 12 in the emergency department reduced the risk for undertriage compared to RTS $=12$ (normal value). Field RTS was documented by anaesthetists in $64 \%$ of the patients compared to $33 \%$ among paramedics.

Patients subject to undertriage had an ISS-adjusted Odds Ratio for 30-day mortality of 2.34 ( $95 \% \mathrm{Cl} \mathrm{I.6-3.4,} \mathrm{p}<0.00 \mathrm{I}$ ) compared to those correctly triaged to TTA.

Conclusion: Triage precision had not improved after TTA guideline introduction. Anaesthetists perform precise trauma triage, whereas paramedics have potential for improvement. Skewed mission profiles makes comparison of differences in triage precision difficult, but criteria or the use of them may contribute. Massive undertriage among paramedics is of grave concern as patients exposed to undertriage had increased risk of dying. 


\section{Background}

Regional trauma care with designated trauma centres improve outcome for trauma patients [1-6]. Essential for these systems is field triage that identifies trauma victims with injury severity that justifies access to the documented benefits of trauma team resuscitation [7]. Some mistriage is unavoidable, given the evolutionary nature of symptoms following major trauma and that field triage is often performed in the early stages of care. Although physician input is known to increase triage precision [8,9], triage is often performed independently by paramedics with limited training in patient evaluation and structured triage decision-making. Imprecise field triage results in overtriage (trauma team activation (TTA) for the minimally injured patient) and undertriage (severely injured patient admitted without TTA). Priority has been to minimize undertriage, as it may result in adverse patient outcome due to denial of the potential benefits of immediate expert assessment and resuscitation provided by the trauma team. Although overtriage does not directly reduce patient safety, it results in overutilization of limited financial and human resources [10-12] and can cause reduced local emergency medical service (EMS) coverage [13]. As with any test, the cost of improved specificity will be reduced sensitivity. American College of Surgeons, Committee on Trauma (ACS-COT) [14] therefore describes 5\% undertriage as acceptable and associated with an overtriage rate of $25 \%-50 \%$.

A wide range of trauma triage criteria have been proposed $[2,5,15-17]$, but there is no consensus on the ultimate set of variables due to local variations in patient severity mix and trauma care organization. Still, many systems have partly adopted criteria proposed by ACS-COT [14], which focus on physiologic, anatomic and mechanistic parameters in addition to comorbidity. Although some of these criteria have been validated as predictors of severe injury [18-23] the majority remains without scientific evidence.

Ulleval University Hospital (UUH) is the largest trauma hospital in Norway and the trauma referral centre for half of the Norwegian population. Previously, UUH lacked a trauma triage protocol, and TTA was based on clinical judgment alone. In the year 2000, an analysis [9] found that the informal TTA system was imprecise with an undertriage of $11 \%$ and overtriage of $58 \%$ for primary admitted patients. Further, field triage was significantly more correct for patients admitted by anaesthetistmanned units than by paramedic-manned ambulances. This revealed an opportunity for improvement that catalysed the introduction of trauma triage guidelines (Fig. 1).

The continuous process of performance improvement as proposed by ACS-COT [14] refers to a cycle of monitoring, finding, fixing, and monitoring again. In order to close the loop, we wanted to describe triage precision among paramedics and anaesthetists after the introduction of the UUH TTA protocol. We also wanted to analyse how age, gender, category of prehospital care provider, vital signs, type of injury and triage criteria influenced triage precision.

\section{Methods Clinical background}

UUH is the major trauma hospital for 550000 and referral trauma hospital for 2.5 million people. The trauma team is one-tiered, with activation procedures partly based on guidelines published by ACS-COT (Fig. 1) [14]. Prehospital EMS units do not activate the trauma team directly, but report their findings to the ambulance dispatch centre. This information is immediately passed on to the nurse coordinator in the emergency department (ED) who activates the trauma team when at least one of four TTA criteria categories is fulfilled (Fig. 1). When in doubt, the nurse coordinator confers with the trauma team leader before TTA. Prehospital emergency care is provided by ordinary ambulance units staffed with paramedics and by anaesthetist-manned ground and air ambulances.

\section{Patients}

We performed a retrospective analysis of prospectively collected data from the UUH trauma registry. The UUH trauma registrar utilizes a search engine to localize all patients with International Classification of Diseases (ICD) S- and T-codes from the hospital administrative system. This list is manually searched for relevant patients (see Fig. 2 for trauma registry inclusion and exclusion criteria). The study was exempted from the demand of informed consent due to anonymity of extracted data and the absence of any treatment study protocol, and the Regional Committee for Research Ethics and the Data Protection Official deemed approval as not necessary.

We included patients admitted to UUH during the period from $1^{\text {st }}$ of January 2001 to $31^{\text {st }}$ of December 2007, included in UUH trauma registry, and assigned one or more AIS codes (AIS 98; Abbreviated Injury Scale, 1990 Revision, Update 98) with an activated trauma team and/ or severe injury. Patients were classified as severely injured if they fulfilled one of the following criteria: Injury Severity Score [24] (ISS) > 15; penetrating trauma to the head, neck, trunk, or extremities proximal to elbow or knee irrespective of ISS; need of intensive care for more than two days; transferred to another hospital intubated within two days; dead from trauma within 30 days. Interhospital transfers to UUH and patients transported by non-healthcare personnel were excluded, as they were not subject to UUH field triage guidelines.

30 days mortality was determined by information from the Norwegian Population Registry and hospital records 


\section{Anatomic Category}

Stab or gunshot wound to head, neck, torso or body proximal to elbow or knee.

Obvious massive haemorrhage.

Obvious massive blunt injury.

Dislocated injury to the pelvis.

Two large fractures.

Flail chest.

Burns $>15 \%$ body surface and/or inhalation damage.

Children, elderly and patients suffering from chronic diseases tolerate less and shall have a lower threshold for TTA.

\section{Physiologic category}

Disturbed respiration: Dyspnoea, tachypnoea or bradypnoea.

Hypotension.

Significantly reduced consciousness.

Trauma team is activated when a stable patient turns unstable.

\section{Mechanism of Injury category}

Co passenger dead.

Trapped in wreck.

Considerable deformation of vehicle compartment.

Ejected from vehicle.

Pedestrian thrown up on vehicle or through the air.

Child hit by vehicle $>30 \mathrm{~km} / \mathrm{h}$.

Fall from $>5$ meter.

If time from accident to assessment is long and the patient seems unaffected, trauma team shall not be activated, but team leader shall be informed of acute injury.

\section{Multiple patients}

$>2$ injured patients admitted simultaneously

UUH: Ulleval University Hospital; TTA: Trauma Team Activation

Figure I

Ulleval University Hospital trauma team activation (TTA) criteria. 


\begin{tabular}{|c|c|}
\hline $\begin{array}{l}\text { Absolute Criteria: } \\
\text { - Activated trauma team } \\
\text { - Penetrating injury to: } \\
\circ \text { Head } \\
\circ \text { Neck } \\
\circ \text { Trunk } \\
\circ \quad \text { Extremities proximal to } \\
\text { knee or elbow } \\
\text { Relative criterion: } \\
\text { ISS } \geq 10\end{array}$ & $\begin{array}{l}\text { Excluded are patients with: } \\
\text { - Isolated fracture and skin injury } \\
\text { (AIS 1) in: } \\
\circ \quad \text { Upper extremity } \\
\circ \text { Lower extremity } \\
\quad \text { Floor of orbita } \\
\text { - Chronic subdural haematoma } \\
\text { - Drowning, inhalation injury, } \\
\text { asphyxia related injury } \\
\text { (hanging, strangulation) } \\
\text { - Secondary admission to UUH } \\
>24 \text { hours after injury }\end{array}$ \\
\hline
\end{tabular}

Figure 2

Inclusion and exclusion criteria for the UUH trauma registry.

[25]. Repatriated foreign citizens with inaccessible information on 30 days survival status were coded as survivors [26].

In patients who were prehospitally intubated and in general anaesthesia on hospital arrival, respiratory rate and Glasgow Coma Scale [27] (GCS) were scored according to values documented by the prehospital services immediately before intubation. In the absence of this information, we estimated the Triage - Revised Trauma Score (RTS) [28] category (0-4) of the variables respiratory rate and GCS score from the patient record, always utilizing the least pathological value when in doubt. In cases with complete lack of information, normal values were used as default [29].

\section{Statistical analysis}

We assumed severely injured patients to potentially benefit from trauma team presence upon admission, and our evaluation of diagnostic precision of triage was based on this assumption. Various parameters can describe trauma triage precision. We defined " "Sensitivity" as the fraction of severely injured patients that were met by a trauma team (Table 1). "Undertriage" was defined as the contrary event, i.e. 1-sensitivity, interpreted as the probability of not being met by a trauma team despite being severely injured. To calculate specificity and thereby the classical definition of overtriage (1-specificity) [30], the number of patients with minor injuries admitted without TTA must be identified. As UUH each year receives a large number

Table I: Injury severity and trauma team activation (TTA)

\begin{tabular}{cccc}
\hline & Severely injured & Not severely injured & Total \\
\hline TTA & $(\mathrm{a})$ & $(\mathrm{b})$ & $(\mathrm{a}+\mathrm{b})$ \\
No TTA & $(\mathrm{c})$ & $(\mathrm{d})$ & $(\mathrm{c}+\mathrm{d})$ \\
\hline Total & $(\mathrm{a}+\mathrm{c})$ & $(\mathrm{b}+\mathrm{d})$ & $(\mathrm{n})$ \\
\hline
\end{tabular}

Sensitivity $=a /(a+c)$; Specificity $=d /(b+d)$

Positive predictive value (PPV) $=\mathrm{a} /(\mathrm{a}+\mathrm{b})$

Undertriage $=\mathrm{I}-$ Sensitivity $=\mathrm{c} /(\mathrm{a}+\mathrm{c})$; Overtriage $=\mathrm{I}-\mathrm{PPV}=\mathrm{b} /(\mathrm{a}+$ b) 
of primary admitted injured patients, the classical definition is of limited value. This sizeable and not easily definable group of patients is seldom considered for TTA, and would strongly bias the calculation of overtriage based on specificity. Optimal utilization of hospital resources requires a triage protocol that excludes minimally injured patients from TTA. Thus, "overtriage" was defined as the complement of the positive predictive value (1-PPV), where PPV represents the probability of a patient being severely injured when the trauma team is activated (Table 1) $[9,31]$. The null hypothesis that the TTA protocol did not improve triage precision was adopted. All data were analyzed using Statistical Package for the Social Sciences, v. 16.0 (SPSS, Inc., Chicago, IL). Data distributions are reported by medians and interquartile ranges (IQR). Nonparametric data were analysed with the Mann-Whitney test. For categorical data, the chi-square test was used and results are reported as odds ratios (OR) with 95\% confidence interval $(95 \% \mathrm{CI})$. We specifically wanted to study undertriage among severely injured patients, therefore undertriage was used as the dependent variable in the univariate and multivariate analyses. We used logistic regression to estimate the adjusted effects of each significant variable from the univariate analysis [31]. Variables were age, handled as a three level categorical variable $(<55$, $55-70,>70$ years), whereas fall (yes, no), prehospital care provider (paramedic, anaesthetist), RTS $(12,<12)$ and gender were handled as dichotomous variables. ISS was handled as numerical value. Statistical significance was assumed for $\mathrm{p}<0.05$.

\section{Results \\ Descriptive}

During the study period, 4885 patients were entered in the UUH trauma registry, of which 4659 fulfilled our study inclusion criteria. Of the included patients, 4208 $(90 \%)$ had suffered blunt and $451(10 \%)$ penetrating injuries as the dominant type of injury. Forty-two patients $(1 \%)$ suffered both penetrating and blunt injury. Median age of included patients was 32 years (IQR $21-47$ ), and median ISS was 9 (IQR $4-21$ ).

\section{Clinical details of severely injured patients}

Of the 4659 patients, 2221 (48\%) fulfilled our criteria for being severely injured. A majority of these, 1662 (75\%), were men. Median ISS was 21 (IQR 14 - 29), with women having significantly higher ISS than men (median ISS 22 vs. $21, p=0.002$ ). Median age was 36 years (IQR $23-53$ ), with a significant difference in median age between the genders (women median 40 vs. men $34, \mathrm{p}<0.001$ ).

\section{Precision in field triage}

Among the 4659 patients included, we recorded 4440 $(95 \%)$ activations of the trauma team. It was not activated for 219 of the 221 severely injured patients; an undertriage of $10 \%$. The team was activated for minor injuries 2 438 times; an overtriage of 55\%. Patients admitted by anaesthetist-manned units had 2\% undertriage (among 1 059 severely injured patients, 25 received no TTA) and $35 \%$ overtriage ( 1598 TTA where 564 were for minor injuries). Patients brought in by paramedics were subject to $17 \%$ undertriage (among 1162 severely injured patients, 194 received no TTA) and 66\% overtriage ( 2842 TTA where 1874 were for minor injuries) (Table 2). Among the 1508 patients with TTA due to the mechanism of injury (MOI) criterion, 392 (26\%) were severely injured (Table 3). The MOI criterion was used for 1052 (37\%) patients admitted by paramedics, compared to 456 (29\%) of those admitted by anaesthetists (Table 4).

\section{Factors associated with undertriage}

Among the 2221 severely injured patients, age was significantly associated with undertriage, with an adjusted odds ratio (OR) of 2.19 for those between $55-70$ years of age (CI $1.45-3.31 ; \mathrm{p}<0.001$ ) compared to those younger than 55 years. For those older than 70 years, adjusted OR for being undertriaged was 5.41 (CI $3.60-8.13$; p < $0.001)$.

Gender per se was also associated with undertriage, with an OR of 1.91 (CI $1.43-2.56$; p < 0.001) for women compared to men. This difference lost its significance when we adjusted for age, giving an OR of 1.25 for women (CI 0.89 - 1.77; $\mathrm{p}=0.202$ ), as females were strongly represented among those over 55 years of age. Admittance by paramedics was also significantly associated with undertriage with an adjusted OR of 5.84 (CI $3.73-9.13 ; \mathrm{p}<0.001$ ) compared to admittance by anaesthetists. Further, fall was associated with undertriage, with an adjusted OR of 4.89 (CI $3.51-6.83 ; \mathrm{p}<0.001$ ). Finally, a Triage - RTS $<12$ in

Table 2: Field triage precision by category of prehospital care before and after introduction of TTA protocol

\begin{tabular}{lccc}
\hline & Without TTA protocol (1996) & With TTA protocol (200I - 2007) & Undertriage \\
\cline { 2 - 3 } & Overtriage & Undertriage & Overtriage \\
\hline All patients & $58 \%$ & $11 \%$ & $55 \%$ \\
Anaesthetist admitted & $44 \%$ & $6 \%$ & $35 \%$ \\
Paramedic admitted & $67 \%$ & $17 \%$ & $66 \%$ \\
\hline
\end{tabular}


Table 3: Association and number of patients by category of prehospital care provider, TTA criteria, undertriage and correct triage

\begin{tabular}{|c|c|c|c|c|c|c|}
\hline & Total & Severely injured & Dead within 30 days & $\begin{array}{c}\text { Proximal penetrating } \\
\text { injury }\end{array}$ & $\begin{array}{l}\mathrm{ICU}>2 \text { days or } \\
\text { transferred intubated }\end{array}$ & ISS $>$ I5 \\
\hline \multicolumn{7}{|l|}{ Admission: } \\
\hline Anaesthetist & I 623 (35\%) & I 059 (65\%) & 185 (1 I\%) & $80(5 \%)$ & $756(47 \%)$ & $902(56 \%)$ \\
\hline Paramedic & 3036 (65\%) & I 162 (38\%) & $173(6 \%)$ & $372(12 \%)$ & $476(16 \%)$ & 739 (24\%) \\
\hline Total & $4659(100 \%)$ & 2221 (48\%) & $358(8 \%)$ & 452 (10\%) & I 232 (26\%) & I $64 \mid$ (35\%) \\
\hline Patients with TTA & 4440 (95\%) & 2002 (45\%) & $316(7 \%)$ & $426(10 \%)$ & I I54 (26\%) & I 467 (33\%) \\
\hline \multicolumn{7}{|l|}{ TTA criteria: } \\
\hline Anatomic & I 192 (27\%) & 702 (59\%) & 107 (9\%) & 235 (20\%) & $36 \mathrm{I}(30 \%)$ & $452(38 \%)$ \\
\hline Physiologic & $76(2 \%)$ & $42(55 \%)$ & $9(12 \%)$ & $12(16 \%)$ & $20(26 \%)$ & $28(37 \%)$ \\
\hline $\mathrm{MOI}$ & I 508 (34\%) & $392(26 \%)$ & $33(2 \%)$ & $4(0 \%)$ & $245(16 \%)$ & $324(22 \%)$ \\
\hline Multiple patients & $8(0 \%)$ & $3(38 \%)$ & $0(0 \%)$ & I (13\%) & I (I3\%) & $2(25 \%)$ \\
\hline Several & $760(17 \%)$ & $504(66 \%)$ & $127(17 \%)$ & $62(8 \%)$ & $351(46 \%)$ & $430(57 \%)$ \\
\hline Unknown & $896(20 \%)$ & $359(40 \%)$ & 40 (5\%) & $112(13 \%)$ & $176(20 \%)$ & $231(26 \%)$ \\
\hline Undertriage & 219 & $219(100 \%)$ & 42 (19\%) & $26(12 \%)$ & 78 (36\%) & $174(80 \%)$ \\
\hline Correct triage & 2002 & $2002(100 \%)$ & $316(16 \%)$ & 426 (21\%) & I $154(58 \%)$ & I $467(73 \%)$ \\
\hline
\end{tabular}

ICU: Intensive Care Unit; ISS: Injury Severity Score; MOI: Mechanism of Injury

the ED reduced the risk for undertriage with an adjusted OR of 0.42 (CI $0.30-0.60 ; \mathrm{p}<0.001)$ compared to RTS = 12 (normal value). Field RTS was documented by anaesthetists in $64 \%$ of the patients compared to $33 \%$ among paramedics $(\mathrm{p}<0.001)$. Factors associated with undertriage are outlined in Table 5.

\section{The consequence of undertriage}

Patients subject to undertriage had significantly higher mortality risk compared to those correctly triaged, with an OR adjusted for ISS of 2.34 (CI $1.59-3.43$; p < 0.001) (Table 6).

\section{Discussion}

Patients brought to UUH by anaesthetists had a satisfactory triage precision, with an undertriage of $2 \%$ and overtriage of $35 \%$, whereas patients brought in by paramedics were subject to unacceptable mistriage, with an undertriage of $17 \%$ and overtriage of $66 \%$ (Table 2 ).

Although patients admitted by paramedics were associated with less injury severity compared to those admitted by anaesthetists (median ISS 5 vs. 17, p < 0.001) due to overtriage, they were subject to a significantly higher risk for undertriage (Table 5). These results indicate that both patients and the trauma system could profit from integrating the highest level of medical competence accessible into the triage process. However, comparison of these patient groups must be made with caution, as skewed mission profiles might contribute to the observed differences.

The overall trauma triage system performance at UUH was outside the recommendations stated in the ACS-COT guidelines $[14]$, with an undertriage of $10 \%$ and overtriage of $55 \%$ (Table 2). However, comparison of triage

Table 4: Usage and performance of TTA criteria by category of prehospital care provider

\begin{tabular}{|c|c|c|c|c|c|c|}
\hline \multirow[b]{2}{*}{ TTA criteria } & \multicolumn{3}{|c|}{ Paramedic } & \multicolumn{3}{|c|}{ Anaesthetist } \\
\hline & Total & Correct triage & Overtriage & Total & Correct triage & Overtriage \\
\hline Anatomic & $7 \mid 7$ (25\%) & 372 (52\%) & 345 (48\%) & 475 (30\%) & $330(70 \%)$ & 145 (30\%) \\
\hline Physiologic & $65(2 \%)$ & $33(51 \%)$ & $32(49 \%)$ & I I (0\%) & $9(82 \%)$ & $2(18 \%)$ \\
\hline $\mathrm{MOI}$ & I 052 (37\%) & $163(15 \%)$ & $889(85 \%)$ & $456(29 \%)$ & $229(50 \%)$ & $227(50 \%)$ \\
\hline Multiple patients & $6(0 \%)$ & $2(33 \%)$ & $4(67 \%)$ & $2(0 \%)$ & I (50\%) & I (50\%) \\
\hline Several criteria & $354(13 \%)$ & $182(51 \%)$ & $172(49 \%)$ & $406(25 \%)$ & $322(79 \%)$ & $84(21 \%)$ \\
\hline No documented criteria & $648(23 \%)$ & $216(33 \%)$ & $432(67 \%)$ & $248(16 \%)$ & 143 (58\%) & $105(42 \%)$ \\
\hline Total & 2842 & 968 (34\%) & I 874 (66\%) & I 598 & I 034 (65\%) & 564 (35\%) \\
\hline
\end{tabular}

MOI: Mechanism of Injury 
Table 5: Triage outcome split by factors associated with undertriage among $222 \mathrm{I}$ severely injured patients. Unadjusted and adjusted (for gender, age, category of prehospital care, ED-RTS and fall), estimates of odds ratio for undertriage with $95 \% \mathrm{Cl}$ and $\mathrm{p}$ values

\begin{tabular}{|c|c|c|c|c|}
\hline & Correct triage $(n=2002)$ & Undertriage $(n=219)$ & OR $(95 \% \mathrm{Cl})$ & Adjusted OR $(95 \% \mathrm{Cl})$ \\
\hline \multicolumn{5}{|l|}{ Gender: } \\
\hline Men & I 525 (76\%) & 137 (63\%) & 1.00 & 1.00 \\
\hline Women & $477(24 \%)$ & $82(37 \%)$ & $1.91(1.43-2.56)^{*}$ & $1.25(0.89-1.77) \dagger$ \\
\hline \multicolumn{5}{|l|}{ Age: } \\
\hline$<55$ years & I 595 (80\%) & 99 (45\%) & 1.00 & 1.00 \\
\hline $55-70$ years & $261(13 \%)$ & $46(21 \%)$ & $2.84(1.96-4.13)^{*}$ & $2.19(1.45-3,31)^{*}$ \\
\hline$>70$ years & $146(7 \%)$ & $74(34 \%)$ & $8.17(5.78-11.54)^{*}$ & $5.41(3.60-8.13)^{*}$ \\
\hline \multicolumn{5}{|l|}{ Admitted by: } \\
\hline Anaesthetist & I $034(52 \%)$ & 25 (11\%) & 1.00 & 1.00 \\
\hline Paramedic & $968(48 \%)$ & $194(89 \%)$ & $8.29(5.42-12.69)^{*}$ & $5.84(3.73-9.13)^{*}$ \\
\hline \multicolumn{5}{|l|}{ ED-RTS: } \\
\hline 12 & I 035 (52\%) & I56 (7I\%) & 1.00 & 1.00 \\
\hline$<12$ & 967 (48\%) & $63(29 \%)$ & $0.43(0.32-0.59)^{*}$ & $0.42(0.30-0.60)^{*}$ \\
\hline \multicolumn{5}{|l|}{ Fall: } \\
\hline No & I $632(82 \%)$ & 81 (37\%) & 1.00 & 1.00 \\
\hline Yes & $370(18 \%)$ & $138(63 \%)$ & $7.52(5.59-10.11)^{*}$ & $4.89(3.5 \mathrm{I}-6.83)^{*}$ \\
\hline
\end{tabular}

Cl: Confidence Interval; OR: Odds Ratio; *: $p<0.001 ; \dagger: p=0.202$

ED-RTS: Revised Trauma Score in the Emergency Department

rates must be made with care, as different definitions of what constitutes a suitable patient for TTA - frequently referred to as a "severely injured" patient - are applied. Injury severity is a continuum and the cut off has traditionally been arbitrary. Nevertheless, the definition is fundamental, as it determines the threshold for inclusion to the care given by an activated trauma team, and provides the retrospective standard against which the triage guidelines will be tested. The US Major Trauma Outcome Study [32] found that ISS > 15 was related to a mortality risk of at least $10 \%$, and despite some well-documented limitations $[33,34]$, this cut off has been widely applied to define severe injury. We addressed these limitations by including proximal penetrating injury, need for ICU care and death from trauma within 30 days [25]. To achieve comparability with a previous analysis [9], the need for urgent ED procedure or operative intervention $[35,36]$ (e.g. damage control laparotomy) was excluded from our definition, highlighting that consensus among researchers regarding a common definition of "severely injured" is needed. The current study is a retrospective review of trauma registry data and as such has several limitations. It is subject to retrospective bias and incomplete data collection, and it is restricted to variables already defined in the trauma registry. Some of the predefined data points (e.g. TTA criteria) lack detail and thus limit analysis precision. Further, the seven years delay between guideline introduction and the study of its efficacy may be considered too long.

Patients admitted by ordinary ambulances were more frequently triaged to TTA due to MOI (Table 4). MOI criteria were generally unable to predict severe injury regardless of personnel category involved in the triage process (Table 3). MOI was introduced as criterion after retrospective studies [37-39] revealed that some blunt trauma scenarios

Table 6: 30 day mortality by category of triage. Unadjusted and adjusted for ISS

\begin{tabular}{|c|c|c|c|c|c|c|}
\hline & \multirow[b]{2}{*}{ Total } & \multicolumn{5}{|c|}{ Dead within 30 days } \\
\hline & & Number of patients & OR $(95 \% \mathrm{Cl})$ & $\mathrm{P}$-value & Adjusted OR $(95 \% \mathrm{Cl})$ & Adjusted $p$-value \\
\hline Correct triage & 2002 & $316(16 \%)$ & 1.00 & & 1.00 & \\
\hline Undertriage & 219 & $42(19 \%)$ & I.27 $(0.89-\mathrm{I}, 8 \mathrm{I})$ & $P=0.23$ & $2.34(1.59-3.43)$ & $P<0.001$ \\
\hline
\end{tabular}

OR: Odds Ratio; Cl: Confidence Intervals; ISS: Injury Severity Score 
were associated with significant victim injury, which might remain occult throughout the prehospital period. Although it was recognized that this criterion would yield over-utilization of trauma centre resources, a certain amount of overtriage was deemed necessary to avoid preventable trauma deaths [14]. Car safety design and the utilization of safety restraints has markedly improved since many of these studies were published, and other papers now confirm the association between MOI as single criterion for TTA and overtriage [13,40-43].

Our results are consistent with prior studies that show that physiological and anatomical trauma triage criteria are predictive of the need for TTA $[13,18,19,21,23]$ (Table 3 ). In general, anaesthetists put more emphasis on vital signs, as evidenced by prehospital RTS [28] being documented for $64 \%$ of the patients compared to $33 \%$ among paramedics ( $\mathrm{p}<0.001)$. Unsurprisingly triage - RTS $<12$ in the ED reduced the risk for undertriage (Table 5). The presence of abnormal vital signs after involvement in trauma may suggest significant haemorrhage and the need for evaluation by the trauma team. However, the absence of abnormal vital signs or obvious anatomic injury does not rule out severe injury. We believe that "physiologic derangement" and "anatomic injury" categories should be mandatory criteria for full TTA at UUH, whereas MOI and "comorbidity" should be downgraded to only activate a trauma team consisting of fewer members. In an attempt to deal with the burden of overtriage generated by excessive use of the MOI criterion several trauma centers have introduced tiered triage systems, and published their positive experiences with them $[11,16,44-46]$.

Patients subject to undertriage had significantly higher mortality risk compared to those correctly triaged, when adjusted for injury severity (Table 6). Phillips and coworkers [47] described falls as the main aetiology behind severe injury among elderly (hip fractures were excluded form the study), and that triage criteria according to ACSCOT recommendations failed to identify these trauma victims. We found both falls and increasing age to be significantly associated with undertriage, but there was no significant difference between genders when adjusted for age (Table 5). Problems in the initial evaluation of the traumatized geriatric patient may contribute to an increased risk of undertriage. Misleadingly "normal" initial vital signs despite severe injury due to medication and an inability to launch normal physiologic responses have been suggested as contributing factors [22]. Elderly trauma patients have particularly high mortality, even with fairly minor or moderately severe injuries. Undertriage in this group probably contributes to an even higher mortality. Demetriades et al. [22] have suggested that age over 70 years alone should be a criterion for TTA. In a later paper, Demetriades and coworkers [48] found that activated trauma team and early intensive monitoring, evaluation, and resuscitation of geriatric trauma patients improved survival.

The present study was conceived to highlight the supposed advantages of a trauma triage protocol, but increased precision could only be demonstrated among anaesthetists (Table 2). Although the introduced guidelines were based on fairly well documented material [18$22]$, triage precision among paramedics did not improve and therefore camouflaged any possible benefit on total system precision. Further, we found examples of breeched guidelines such as EMS providers activating the trauma team from the field instead of via the trauma coordinator. Such failure of guideline adherence may also contribute to this unexpected lack of increased triage precision. These results indicate that paramedics need further training in evaluating trauma victims. We also call for improved routines in communicating patient data from EMS units to the nurse coordinator in the $\mathrm{ED}$, with vital signs, obvious anatomic injury, injury mechanism and comorbidity to be ordinal reported. Further, nurse coordinators would benefit from additional training in triage decision-making.

\section{Conclusion}

Evaluating vital signs and anatomic injury require competence, and anaesthetists performed field triage with higher precision than paramedics, who displayed an unacceptably high mistriage rate. We therefore failed to reject the null hypothesis about any benefit brought about by introducing a trauma triage protocol. The discrepancy between personnel categories amplifies the need for a user-friendlier triage protocol and increased competence in trauma patient evaluation among paramedics. Although MOI with its low prediction accuracy was extensively used as TTA criterion, this alone could not explain all the imprecision. The "physiologic" and "anatomic" criteria performed well. Our findings should be an incitement to design a two-tiered trauma triage protocol, and thereafter change provider behaviour through a well-documented implementation strategy.

\section{Competing interests}

The authors declare that they have no competing interests.

\section{Authors' contributions}

MR and HML conceived the study. MR, TE, AJK, NOS and HML designed the study. MR and AJK performed the data analysis. NOS and TE designed and developed the UUH trauma registry. MR drafted the manuscript. All authors interpreted data and critically revised the manuscript. All authors have read and approved the final manuscript 


\section{Acknowledgements}

We thank UUH Trauma registrar Morten Hestnes for valuable comments on data variables.

The Norwegian Air Ambulance Foundation and Health Region Southeast provided funding.

\section{References}

I. West JG, Cales RH, Gazzaniga AB: Impact of regionalization. The Orange County experience. Archives of Surgery 1983, I I 8:740-744.

2. Shackford SR, Hollingworth-Fridlund P, Cooper GF, Eastman AB: The effect of regionalization upon the quality of trauma care as assessed by concurrent audit before and after institution of a trauma system: a preliminary report. Journal of TraumaInjury Infection \& Critical Care 1986, 26:812-820.

3. Liberman M, Mulder DS, Lavoie A, Sampalis JS: Implementation of a trauma care system: evolution through evaluation. Journal of Trauma-Injury Infection \& Critical Care 2004, 56: I 330-I 335.

4. Mullins RJ, Veum-Stone J, Hedges JR, Zimmer-Gembeck MJ, Mann NC, Southard PA, Helfand M, Gaines JA, Trunkey DD: Influence of a statewide trauma system on location of hospitalization and outcome of injured patients. Journal of Trauma-Injury Infection \& Critical Care 1996, 40:536-545. discussion 545-536

5. Kilberg L, Clemmer TP, Clawson J, Woolley FR, Thomas F, Orme JF Jr: Effectiveness of implementing a trauma triage system on outcome: a prospective evaluation. Journal of Trauma-Injury Infection \& Critical Care 1988, 28: |493-| 498.

6. MacKenzie EJ, Rivara FP, Jurkovich G], Nathens AB, Frey KP, Egleston BL, Salkever DS, Scharfstein DO: A national evaluation of the effect of trauma-center care on mortality. New England Journal of Medicine 2006, 354:366-378.

7. Petrie D, Lane P, Stewart TC: An evaluation of patient outcomes comparing trauma team activated versus trauma team not activated using TRISS analysis. Trauma and Injury Severity Score. Journal of Trauma-Injury Infection \& Critical Care 1996, 41:870-873, discussion 873-875

8. Champion HR, Sacco WJ, Gainer PS, Patow SM: The effect of medical direction on trauma triage. Journal of Trauma-Injury Infection \& Critical Care 1988, 28:235-239.

9. Lossius H, Langhelle A, Pillgram-Larsen J, Lossius T, Soreide E, Laake $P$, Steen P: Efficiency of activation of the trauma team in a Norwegian trauma referral centre. The European journal of surgery 2000, 166:760-764.

10. Hoff WS, Tinkoff GH, Lucke JF, Lehr S: Impact of minimal injuries on a level I trauma center. J Trauma 1992, 33(3):408-4I2.

II. DeKeyser FG, Paratore A, Seneca RP, Trask A: Decreasing the cost of trauma care: a system of secondary inhospital triage. Annals of Emergency Medicine 1994, 23:84I-844.

12. MacKenzie EJ, Morris JA Jr, Smith GS, Fahey M: Acute hospital costs of trauma in the United States: implications for regionalized systems of care. Journal of Trauma-Injury Infection \& Critical Care 1990, 30(9): 1096-I101.

13. Henry MC, Alicandro JM, Hollander JE, Moldashel JG, Cassara G, Thode HC Jr: Evaluation of American College of Surgeons trauma triage criteria in a suburban and rural setting. $A m J$ Emerg Med 1996, I4(2): I24-129.

14. ACS-COT: Resources for optimal care of the injured patient: 2006. Chicago: American College of Surgeons; 2006.

15. Cook CH, Muscarella P, Praba AC, Melvin WS, Martin LC: Reducing overtriage without compromising outcomes in trauma patients. Archives of Surgery 200I, I 36:752-756.

16. Phillips JA, Buchman TG: Optimizing prehospital triage criteria for trauma team alerts. Journal of Trauma-Injury Infection \& Critical Care 1993, 34:127-132.

17. Lehmann RK, Arthurs ZM, Cuadrado DG, Casey LE, Beekley AC, Martin MJ: Trauma team activation: simplified criteria safely reduces overtriage. American Journal of Surgery 2007, 193:630-634. discussion 634-635

18. Norwood S, McAuley C, Berne J, Vallina V, Creath R, McLarty J: A prehospital glasgow coma scale score $<$ or $=14$ accurately predicts the need for full trauma team activation and patient hospitalization after motor vehicle collisions. Journal of Trauma-Injury Infection \& Critical Care 2002, 53:503-507.
19. Husum H, Gilbert M, Wisborg T, Van Heng Y, Murad M: Respiratory rate as a prehospital triage tool in rural trauma. Journal of Trauma-Injury Infection \& Critical Care 2003, 55:466-470.

20. Sava J, Alo K, Velmahos GC, Demetriades D: All patients with truncal gunshot wounds deserve trauma team activation. Journal of Trauma-Injury Infection \& Critical Care 2002, 52:276-279.

21. Tinkoff $\mathrm{GH}, \mathrm{O}^{\prime}$ Connor RE: Validation of new trauma triage rules for trauma attending response to the emergency department. Journal of Trauma-Injury Infection \& Critical Care 2002, 52: II53-II58. discussion II58-II59

22. Demetriades D, Sava J, Alo K, Newton E, Velmahos G, Murray J, Belzberg $\mathrm{H}$, Asensio J, Berne $\mathrm{T}$ : Old age as a criterion for trauma team activation. Journal of Trauma-Injury Infection \& Critical Care 200I, 5 I:754-756.

23. Franklin G, Boaz P, Spain D, Lukan J, Carrillo E, Richardson J: Prehospital hypotension as a valid indicator of trauma team activation. Journal of Trauma-Injury Infection \& Critical Care 2000, 48: $1034-1037$.

24. Baker SP, O'Neill B, Haddon W Jr, Long WB: The injury severity score: a method for describing patients with multiple injuries and evaluating emergency care. J Trauma 1974, I4(3): I87-196.

25. Skaga NO, Eken T, Jones JM, Steen PA: Different definitions of patient outcome: consequences for performance analysis in trauma. Injury 2008, 39:612-622.

26. Skaga NO, Eken T, Sovik S, Jones JM, Steen PA: Pre-injury ASA physical status classification is an independent predictor of mortality after trauma. J Trauma 2007, 63(5):972-978.

27. Teasdale G, Jennett B: Assessment of coma and impaired consciousness. A practical scale. Lancet I974, 2:8I-84.

28. Champion HR, Sacco WJ, Copes WS, Gann DS, Gennarelli TA, Flanagan ME: A revision of the Trauma Score. Journal of Trauma-Injury Infection \& Critical Care 1989, 29:623-629.

29. Skaga NO, Eken T, Steen PA: Assessing quality of care in a trauma referral center: benchmarking performance by TRISS-based statistics or by analysis of stratified ISS data? Journal of Trauma-Injury Infection \& Critical Care 2006, 60:538-547.

30. Eastman AB, Lewis FR Jr, Champion HR, Mattox KL: Regional trauma system design: critical concepts. American Journal of Surgery 1987, 154:79-87.

31. Altman D: Practical statistics for medical research London, UK: Chapman \& Hall; 1991.

32. Champion HR, Copes WS, Sacco WJ, Lawnick MM, Keast SL, Bain LW Jr, Flanagan ME, Frey CF: The Major Trauma Outcome Study: establishing national norms for trauma care. Journal of Trauma-Injury Infection \& Critical Care 1990, 30:1356-1365.

33. Lossius H, Langhelle A, Soreide E, Pillgram-Larsen J, Lossius T, Laake $P$, Steen P: Reporting data following major trauma and analysing factors associated with outcome using the new Utstein style recommendations. Resuscitation 2001, 50:263-272.

34. Baxt WG, Upenieks V: The lack of full correlation between the Injury Severity Score and the resource needs of injured patients. Ann Emerg Med 1990, I9:1396-1400.

35. Kruger AJ, Hesselberg N, Abrahamsen GT, Bartnes K: [When should the trauma team be activated?]. Tidsskrift for Den Norske Laegeforening 2006, I 26: I 335- I337.

36. Ringdal KG, Coats T], Lefering R, Di Bartolomeo S, Steen PA, Røise $\mathrm{O}$, Handolin L, Lossius HM: The Utstein template for uniform reporting of data following major trauma: a joint revision by SCANTEM, TARN, DGU-TR and RITG. Scand J Trauma Resusc Emerg Med 2008, I 6:7. (28 August 2008)

37. Lowe DK, Oh GR, Neely KW, Peterson CG: Evaluation of injury mechanism as a criterion in trauma triage. American Journal of Surgery 1986, I 52:6-10.

38. Cottington EM, Young JC, Shufflebarger CM, Kyes F, Peterson FV Jr, Diamond DL: The utility of physiological status, injury site, and injury mechanism in identifying patients with major trauma. Journal of Trauma-Injury Infection \& Critical Care 1988, 28:305-3 I I.

39. Jones IS, Champion HR: Trauma triage: vehicle damage as an estimate of injury severity. J Trauma 1989, 29:646-653.

40. Shatney C, Sensaki K: Trauma team activation for 'mechanism of injury' blunt trauma victims: time for a change? Journal of Trauma-Injury Infection \& Critical Care 1994:275-28I.

41. Uleberg O, Vinjevoll OP, Eriksson U, Aadahl P, Skogvoll E: Overtriage in trauma - what are the causes? Acta Anaesthesiologica Scandinavica 2007, 5 I: I | 78- | | 83. 
42. Kann SH, Hougaard K, Christensen EF: Evaluation of pre-hospital trauma triage criteria: a prospective study at a Danish level I trauma centre. Acta Anaesthesiologica Scandinavica 2007, 5I:II72-II77.

43. Clemmesen ML, Rytter S, Birch K, Lindholt JS, Jensen SS, Troelsen S: [Should high-energy traumas always result in a trauma team call?]. Ugeskrift for laeger 2006, 168:2916-2920.

44. Tinkoff GH, O'Connor RE, Fulda GJ: Impact of a two-tiered trauma response in the emergency department: promoting efficient resource utilization. Journal of Trauma-Injury Infection \& Critical Care 1996, 41:735-740.

45. Plaisier BR, Meldon SW, Super DM, Jouriles NJ, Barnoski AL, Fallon WF Jr, Malangoni MA: Effectiveness of a 2-specialty, 2-tiered triage and trauma team activation protocol. Annals of Emergency Medicine I998, 32:436-44I.

46. Ochsner MG, Schmidt JA, Rozycki GS, Champion HR: The evaluation of a two-tier trauma response system at a major trauma center: is it cost effective and safe? Journal of Trauma-Injury Infection \& Critical Care 1995, 39:97I-977.

47. Phillips S, Rond PC 3rd, Kelly SM, Swartz PD: The failure of triage criteria to identify geriatric patients with trauma: results from the Florida Trauma Triage Study. Journal of Trauma-Injury Infection \& Critical Care 1996, 40:278-283.

48. Demetriades D, Karaiskakis M, Velmahos G, Alo K, Newton E, Murray J, Asensio J, Belzberg H, Berne T, Shoemaker W: Effect on outcome of early intensive management of geriatric trauma patients. The British journal of surgery 2002, 89:1319-1322.

Publish with Bio Med Central and every scientist can read your work free of charge

"BioMed Central will be the most significant development for disseminating the results of biomedical research in our lifetime. "

Sir Paul Nurse, Cancer Research UK

Your research papers will be:

- available free of charge to the entire biomedical community

- peer reviewed and published immediately upon acceptance

- cited in PubMed and archived on PubMed Central

- yours - you keep the copyright 